\title{
PACAP Enhances Barrier Properties of Cerebral Microvessels
}

Imola Wilhelm 1

Csilla Fazakas 1

Andrea Tamás 2

Gábor Tóth 3

Dóra Reglődi 2

István A. Krizbai $1, *$

Phone $+36-62-599602$

Fax +36-62-433133

Email krizbai.istvan@brc.mta.hu

1 Institute of Biophysics, Biological Research Centre, Hungarian Academy of Sciences, Szeged, Hungary

2 Department of Anatomy, PTE-MTA “Lendület” PACAP Research Team, University of Pécs, Pécs, Hungary

3 Department of Medical Chemistry, University of Szeged, Szeged, Hungary

\section{Abstract}

Cerebral microvascular endothelial cells-coming in contact with pericytes and astrocytes-constitute the structural basis of the blood-brain barrier (BBB). The continuous belt of interendothelial tight junctions (TJs) and the presence of specific transport systems, enzymes, and receptors in the brain endothelium regulate the molecular and cellular traffic into the central nervous system. Pituitary adenylate cyclase-activating polypeptide (PACAP) is a neuropeptide having several cellular protective effects. However, little is known about the effects of PACAP on the cerebral endothelium and BBB functions. Here, we show that PACAP has no significant pro-survival role in cerebral microvascular endothelial cells; 
however, it improves the barrier properties of the brain endothelium.

PACAP induces an increase in the transendothelial electrical resistance, which is the most important marker of the tightness of the TJs. Moreover, PACAP has a protective role against glucose deprivation- and oxidative stress-induced junctional damage in microvascular brain endothelial cells.

\section{Keywords}

Cerebral microvascular endothelial cell

Blood-brain barrier (BBB)

Tight junctions

Transendothelial electrical resistance (TEER)

Pituitary adenylate cyclase-activating polypeptide (PACAP)

Cyclic adenosine monophosphate (cAMP)

Imola Wilhelm and Csilla Fazakas contributed equally to this work.

Electronic supplementary material

The online version of this article (doi: 10.1007/s12031-014-0260-4 ) contains supplementary material, which is available to authorized users.

\section{Introduction}

Neurons of the vertebrate brain require strictly regulated concentrations of ions, neurotransmitters, hormones, and other biologically active substances. The blood-brain barrier (BBB) is one of the most important systems responsible for the maintenance of this microenvironment. The $\mathrm{BBB}$ is formed by brain capillary endothelial cells in close association with pericytes and astrocytic end feet almost completely covering the cerebral surface of the endothelium. In comparison to endothelia of non-cerebral origin, brain endothelia undergo an additional step of differentiation which results in a specific cellular phenotype that resembles epithelial cells. In this respect, the most prominent feature of cerebral endothelial cells is the occurrence of continuous tight junctions (TJs) which seal the paracellular passage for macromolecules and cells from the blood stream into the brain parenchyma. Other characteristics specific to cerebral endothelial cells include the absence of fenestrations, the paucity of pinocytotic vesicles, the high number of mitochondria, and a variety of transport systems in the luminal and abluminal cellular membrane (for review see: Abbott et al. 2010; Wilhelm et al. 2011).

Tight junctions regulate the paracellular permeability of the endothelial layer 
and also organize the polarization of the endothelial cell membrane separating an apical and a basolateral surface. By regulating the traffic of ions, tight junctions are responsible for establishing the transendothelial electrical resistance (TEER) between the apical and basolateral side of the cells. Tight junctions are composed of transmembrane proteins, including occludin, claudins (mainly claudin-5), and junctional adhesion molecules; and cytoplasmic plaque proteins which comprise zonula occludens proteins (ZO-1, $\mathrm{ZO}-2$ ) and associated molecules. Formation and maintenance of the TJs are supported by the presence of adherens junctions which are also composed of transmembrane and cytoplasmic proteins (the cadherin-catenin and the nectinafadin systems). The main cadherin of endothelial cells is VE-cadherin.

Pituitary adenylate cyclase-activating polypeptide (PACAP) was originally isolated as a hypophysiotropic neuropeptide (Miyata et al. 1989). Later it was shown that it is broadly expressed in the central nervous system and in several other organs as well, including the retina, kidney, immune cells, reproductive tissues, gastrointestinal tract, etc. PACAP exerts neuroprotective (Tsuchikawa et al. 2012), cytoprotective, pro-survival (Brown et al. 2013), anti-inflammatory, vasodilator, and several other effects (for review see: Vaudry et al. 2009; Reglodi et al. 2012). PACAP has been shown to have several effects on microvascular endothelia of non-cerebral origin: it protects mouse hemangioendothelioma cells against the apoptosis-inducing effects of oxidative stress (Racz et al. 2007) and inhibits hyperglycemia-induced proliferation in $\mathrm{H} 5 \mathrm{~V}$ murine microvascular endothelial cells (Castorina et al. 2010 ). However, the effects of PACAP on brain endothelial cells are incompletely understood. It has been shown that PACAP exerts an inhibitory effect on prostanoid synthesis in rat cerebral microvessels via a cyclic adenosine monophosphate (cAMP)-dependent pathway (Kis et al. 1999). It has also been shown that PACAP crosses the BBB at a modest degree. The influx of PACAP into the brain is different between the two isoforms: PACAP-27 enters the brain by transmembrane diffusion, while PACAP-38 by a saturable transport system (Banks et al. 1993). The peptide transport system-6 (PTS-6) is responsible for the bidirectional transport of PACAP-38 and for the efflux of PACAP-27 (Dogrukol-Ak et al. 2009). On the other hand, it has not been investigated whether PACAP has any effect on the barrier properties of the brain endothelium.

\section{Material and Methods}


The human cerebral microvascular endothelial cell line (hCMEC/D3, shortly D3) (Weksler et al. 2005) was cultured on rat tail collagen-coated surfaces in EBM-2 medium (Lonza) supplemented with $5 \%$ FBS (Lonza) and EGM-2 (Lonza). For immunofluorescence experiments, after reaching confluence, cells were given $10 \mathrm{mM} \mathrm{LiCl}$ for $48 \mathrm{~h}$ (Paolinelli et al. 2013).

Primary rat brain microvascular endothelial cells (RBECs) were isolated from 2-3-week-old Wistar rats, as described previously (Wilhelm et al. 2007; Hutamekalin et al. 2008). Briefly, after removal of meninges, cerebral cortices were cut into small pieces and digested with $1 \mathrm{mg} / \mathrm{ml}$ collagenase type 2 for $90 \mathrm{~min}$ at $37^{\circ} \mathrm{C}$. After separation of myelin by centrifugation in $20 \%$ bovine serum albumin (BSA, Sigma), a second digestion was performed with $1 \mathrm{mg} / \mathrm{ml}$ collagenase/dispase for $50 \mathrm{~min}$ at $37{ }^{\circ} \mathrm{C}$. Microvessel fragments were collected after centrifugation on Percoll gradient (10 min 1,000 g) and plated onto fibronectin/collagen-coated dishes. Endothelial cells growing out of the microvessels were cultured in Dulbecco's Modified Eagle's Medium (DMEM)/F12, $10 \%$ plasma-derived serum (First Link, UK), and growth factors. In the first 2 days, $4 \mu \mathrm{g} / \mathrm{ml}$ puromycin was added to remove contaminating cells. After reaching confluence, the endothelial monolayer was gently trypsinized and passed onto filter inserts (Transwell-Clear, CostarCorning, $1.12 \mathrm{~cm}^{2}, 0.4-\mu \mathrm{m}$ pore size). The next day, the endothelial monolayer was supplied with $550 \mathrm{nM}$ hydrocortisone, $250 \mu \mathrm{M}$ CPT-cAMP, and $17.5 \mu \mathrm{M}$ RO-201724.

Rat astrocytes and pericytes were isolated as described previously (Sziraki et al. 2011 ) and cultured in DMEM (LifeTechnologies) supplemented with $10 \%$ FBS (Lonza).

Confluent RBEC or D3 monolayers were treated in serum-free DMEM or glucose-free DMEM (LifeTechnologies) using 100 nM PACAP1-38 (synthesized at the Department of Medical Chemistry, University of Szeged). For TEER measurements, cells were treated in complete medium, and PACAP was applied either in the apical/upper or in the basolateral/lower or in both chambers. DMNQ (2,3-dimethyl-1,4-naphthoquinone; Sigma) was applied in a concentration of $25 \mu \mathrm{M}$.

\section{RT-PCR}

Total RNA was extracted from untreated cells using TRIzol reagent (LifeTechnologies) following the manufacturer's recommendations. RNA was 
transcribed into complementary (c)DNA using a reverse transcription kit (Thermo Scientific). The amplification was performed on a Bio-Rad iQ5 thermal cycler using Taq polymerase (Roche) under the following conditions: 40 cycles of $95{ }^{\circ} \mathrm{C}$ for $15 \mathrm{~s}, 56{ }^{\circ} \mathrm{C}$ for $30 \mathrm{~s}$, and $72{ }^{\circ} \mathrm{C}$ for $30 \mathrm{~s}$. Forward and reverse primers used for the amplification are listed in Table 1.

Table 1

Primers used

\begin{tabular}{|c|c|c|}
\hline & Sequence $\left(5^{\prime}-3^{\prime}\right)$ & Reference \\
\hline \multicolumn{3}{|c|}{ Rat primers } \\
\hline $\begin{array}{l}\text { rPACAP } \\
\text { fw }\end{array}$ & ATGTCGCCCACGAAATCCTT & \multirow{8}{*}{$\begin{array}{l}\text { Giunta In table } 1 \text { this } \\
\text { reference refers to the last } 4 \text { rows } \\
\text { (rVPAC1 fw, rVPAC1 rv, rVPAC2 fw } \\
\text { and rVPAC2 rv) and not to the } \\
\text { whole set of rat primers. et al. } \\
\mathbf{2 0 1 2}\end{array}$} \\
\hline $\begin{array}{l}\text { rPACAP } \\
\text { rv }\end{array}$ & TGTCTGTGAAGATGCCGTCC & \\
\hline $\begin{array}{l}\text { rPAC1 } \\
\text { fw }\end{array}$ & AAATGCTACTGCAAGCCACA & \\
\hline $\mathrm{rv}^{\mathrm{rPAC} 1}$ & GCTCTTGCTCAGGATGGACA & \\
\hline $\begin{array}{l}\text { rVPAC1 } \\
\text { fw }\end{array}$ & GTGAAGACCGGCTACACCAT & \\
\hline $\begin{array}{l}\text { rVPAC1 } \\
\text { rV }\end{array}$ & TGAAGAGGGCCATATCCTTG & \\
\hline $\begin{array}{l}\text { rVPAC2 } \\
\text { fw }\end{array}$ & AGAGCCATCTCTGTGCTGGT & \\
\hline $\begin{array}{l}\text { rVPAC2 } \\
\text { rv }\end{array}$ & AGGTAGGCCAGGAAACACCT & \\
\hline \multicolumn{3}{|c|}{ Human primers } \\
\hline $\begin{array}{l}\text { hPACAP } \\
\text { fw }\end{array}$ & CGGGAGGAGTTGAAGGAATGA & \multirow{3}{*}{$\begin{array}{l}\text { Basille In table } 1 \text { this } \\
\text { reference refers to the last } 4 \text { rows } \\
\text { (hVPAC1 fw, hVPAC1 rv, hVPAC2 fw } \\
\text { and hVPAC2 rv) and not to the } \\
\text { whole set of human primers. et al. } \\
2006\end{array}$} \\
\hline $\begin{array}{l}\text { hPACAP } \\
\text { rv }\end{array}$ & GACAGCCATTTGTTTCCGGT & \\
\hline $\begin{array}{l}\text { hPAC1 } \\
\text { fw }\end{array}$ & AGCATCTACTTCAGCTGCGTG & \\
\hline
\end{tabular}




\begin{tabular}{|l|l|l|}
\hline $\begin{array}{l}\text { hPAC1 } \\
\text { rv }\end{array}$ & TTCCAGCTTCGCCATTTTCG & Reference \\
\hline $\begin{array}{l}\text { hVPAC1 } \\
\text { fw }\end{array}$ & ATGTGCAGATGATCGAGGTG & \\
\hline $\begin{array}{l}\text { hVPAC1 } \\
\text { rv }\end{array}$ & TGTAGCCGGTCTTCACAGAA & \\
\hline $\begin{array}{l}\text { hVPAC2 } \\
\text { fw }\end{array}$ & CTTCAGGAAGCTGCACTGC & \\
\hline $\begin{array}{l}\text { hVPAC2 } \\
\text { rv }\end{array}$ & CAAACACCATGTAGTGGACG & \\
\hline
\end{tabular}

\section{Determination of Apoptosis}

D3 monolayers were treated in serum-free DMEM or glucose-free DMEM with $25 \mu \mathrm{M}$ DMNQ and/or $100 \mathrm{nM}$ PACAP for $90 \mathrm{~min}$. Apoptotic cells were stained with anti-cleaved caspase 3 antibody (Cell Signaling). Simultaneously with the secondary antibody, $0.6 \mu \mathrm{g} / \mathrm{ml}$ Hoechst-33342 was added for marking the nuclei. The number of apoptotic cells was compared to the total cell number. We have also analyzed the expression profile of apoptosis-related proteins using a human apoptosis array kit (R\&D Systems) according to the manufacturer's instructions.

\section{Measurement of Transendothelial Electrical Resistance}

RBECs were grown on semipermeable filters (as described) and placed into the wells of the cellZscope ${ }^{\circledR}$ instrument (nanoAnalytics). Cells were treated with PACAP when the TEER had reached plateau (i.e., the monolayer has reached total confluence and mature junctions have been formed). TEER was afterwards monitored for $4 \mathrm{~h}$.

\section{Immunofluorescence}

For immunofluorescence studies, cells were cultured on 96-well plates. Brain endothelial monolayers were fixed using a mixture of ice cold ethanol/acetic acid (95:5) for $5 \mathrm{~min}$ and then washed three times for $5 \mathrm{~min}$ in phosphatebuffered saline (PBS). After blocking with $3 \%$ BSA for $30 \mathrm{~min}$, the 
monolayers were incubated with primary antibodies against occludin (LifeTechnologies), claudin-5 (LifeTechnologies), VE-cadherin (Santa Cruz), or ZO-1 (LifeTechnologies). After washing, Cy3- or Alexa488-conjugated secondary antibodies (Jackson ImmunoResearch) were applied, followed by a second washing step. Distribution of the signal was studied using a Nikon Eclipse TE2000U photomicroscope with epifluorescent capabilities connected to a digital camera (Spot RT KE, Diagnostic Instruments).

\section{Western-Blot Analysis}

Cells were washed with PBS and scraped into ice-cold radioimmunoprecipitation assay (RIPA) lysis buffer $(20 \mathrm{mM}$ Tris $(\mathrm{pH}=7.4)$, $150 \mathrm{mM} \mathrm{NaCl}, 1 \%$ sodium deoxycholate, $0.1 \%$ sodium dodecyl sulfate, $1 \mathrm{mM}$ sodium vanadate, $10 \mathrm{mM} \mathrm{NaF}, 1 \mathrm{mM}$ Pefabloc) and incubated on ice for $30 \mathrm{~min}$. Lysates were clarified by centrifugation at 10,000 $\mathrm{g}$ for $10 \mathrm{~min}$ on $4{ }^{\circ} \mathrm{C}$. The protein concentration of the supernatants was determined with the bicinchoninic acid (BCA) method (Pierce). Samples were mixed with Laemmli buffer (final concentration: $12 \mathrm{mM}$ Tris- $\mathrm{HCl}(\mathrm{pH}=6.8), 5 \%$ glycerol, $0.4 \%$ sodium dodecyl sulfate, $14.4 \mu \mathrm{M}$ 2-mercaptoethanol, $0.02 \%$ bromophenol blue) and boiled for 3 min. Proteins were electrophoresed with standard denaturing SDS-PAGE procedures and blotted on nitrocellulose membranes (Protran or Bio-Rad). Blocking the non-specific binding capacity of the membranes was carried out at room temperature for $30 \mathrm{~min}$ in TBS-T (Tris buffered saline with $0.1 \%$ Tween 20 ) containing $3 \%$ BSA or $5 \%$ casein (non-fat milk powder). Blots were then incubated with the primary antibodies. After washing the membranes three times for $10 \mathrm{~min}$ in TBS-T, blots were incubated with the secondary antibodies (Pierce), then washed again three times for $10 \mathrm{~min}$ in TBS-T. The immunoreaction was visualized using Immobilon Western Chemiluminescent HRP Substrate (Millipore) on X-ray film (Agfa). Densitometric analysis was performed using the ImageJ software.

\section{Results}

\section{Characterization of the Expression of PACAP and PACAP Receptors on Cells Forming the BBB}

First we studied the expression of PACAP and its receptors on brain endothelial cells (primary rat brain microvascular endothelial cells and the human microvascular cerebral endothelial cell line D3) using reverse transcriptase polymerase chain reaction (RT-PCR). In order to avoid false positive results originating from genomic contamination, all primer pairs were 
designed from different exons (Table 1). As shown on Fig. 1, we found the presence of PACAP and VPAC2 messenger $(\mathrm{m}) \mathrm{RNA}$ in both types of brain endothelial cells analyzed and VPAC1 in the human endothelial cell line. PAC1 receptor was expressed neither in rat nor in human brain endothelial cells. We have also analyzed the expression of PACAP and PACAP receptors on other cells associated with the BBB. Astrocytes expressed PACAP and all the three receptors, while in pericytes we could detect the mRNA of VPAC2 and low amounts of PAC1 and VPAC1.

\section{Fig. 1}

Expression of PACAP and PACAP receptors on cells of the BBB. RT-PCR was performed using cDNA isolated from primary RBECs, rat astrocytes and pericytes, and the human cerebral endothelial cell line D3. One representative of three independent experiments (in case of primary cells: of three independent cell isolations) is shown. Positive controls: rat brain and human tonsil, negative controls: non-template reactions 


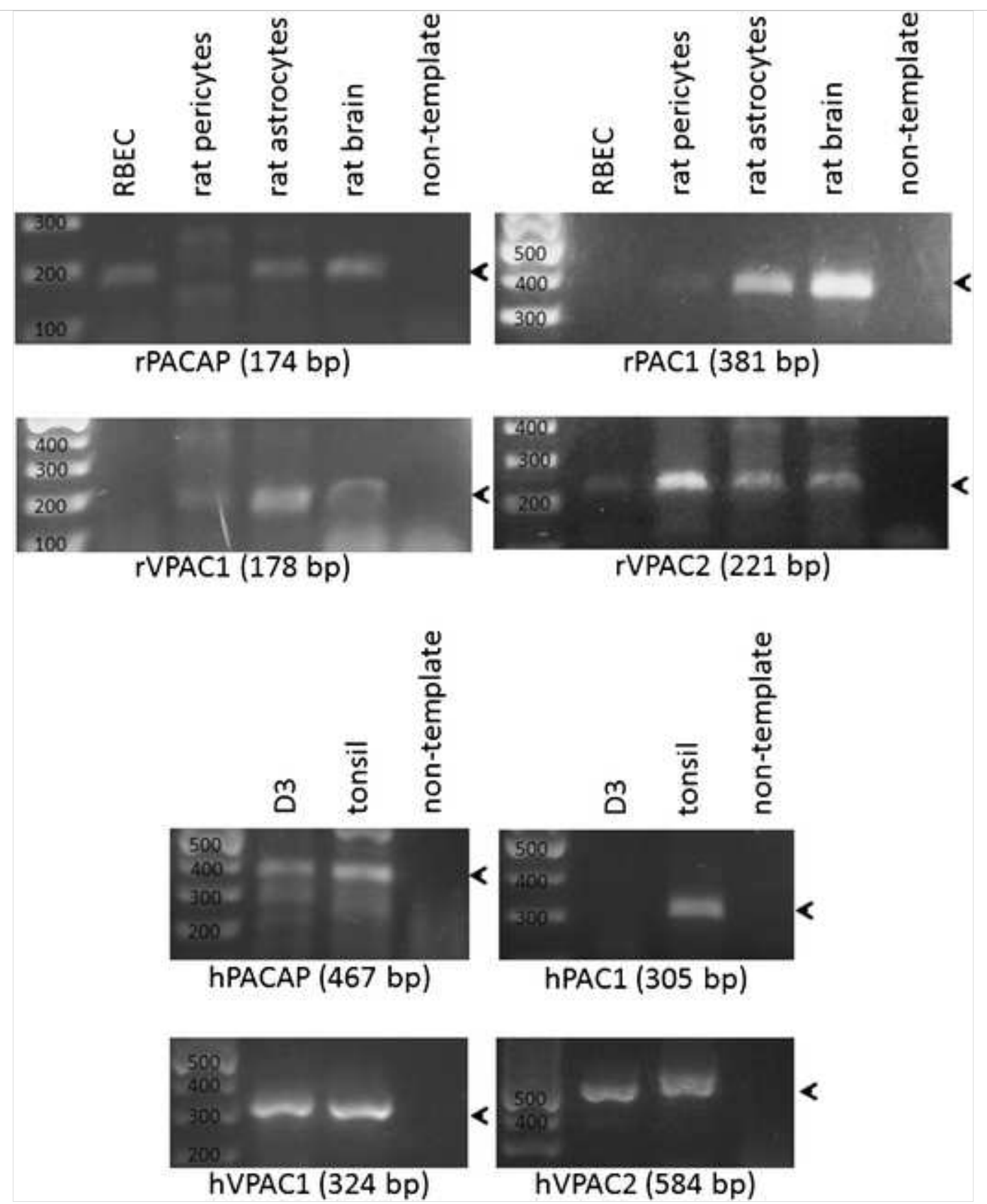

\section{Effect of PACAP on the Survival of Brain Endothelial Cells}

Since PACAP was shown to attenuate apoptosis in mouse hemangioendothelioma (Racz et al. 2007) and several other cell types (Seaborn et al. 2011), we wanted to test whether PACAP also had anti-apoptotic effect in brain endothelial cells. We examined cells exposed to glucose deprivation and/or oxidative stress (using DMNQ) treated or not with 100 nM PACAP1-38. Apoptosis was detected using immunostaining of cleaved caspase 3 positive cells (Fig. 2a) and an antibody array (Fig. 2b and Suppl. Fig. 1B). Although clear morphological changes and cell death were detected (Suppl. Fig. 1A), we have observed only a slight induction of the number of apoptotic cells and apoptotic markers in response to glucose deprivation and oxidative stress. Our results showed that PACAP had no 
significant anti-apoptotic or pro-survival effect in cerebral endothelial cells (Fig. 2 and Suppl. Fig. 1).

Fig. 2

Evaluation of apoptosis in brain endothelial cells. D3 cells were exposed to glucose deprivation- and/or DMNQ-induced oxidative stress in the absence or presence of $100 \mathrm{nM}$ PACAP. a Cleaved caspase 3 positive cells were counted and compared to the total number of nuclei. b Cell lysates were applied onto the human apoptosis array kit. Quantitative analysis of the data is shown on Suppl. Fig. 1B

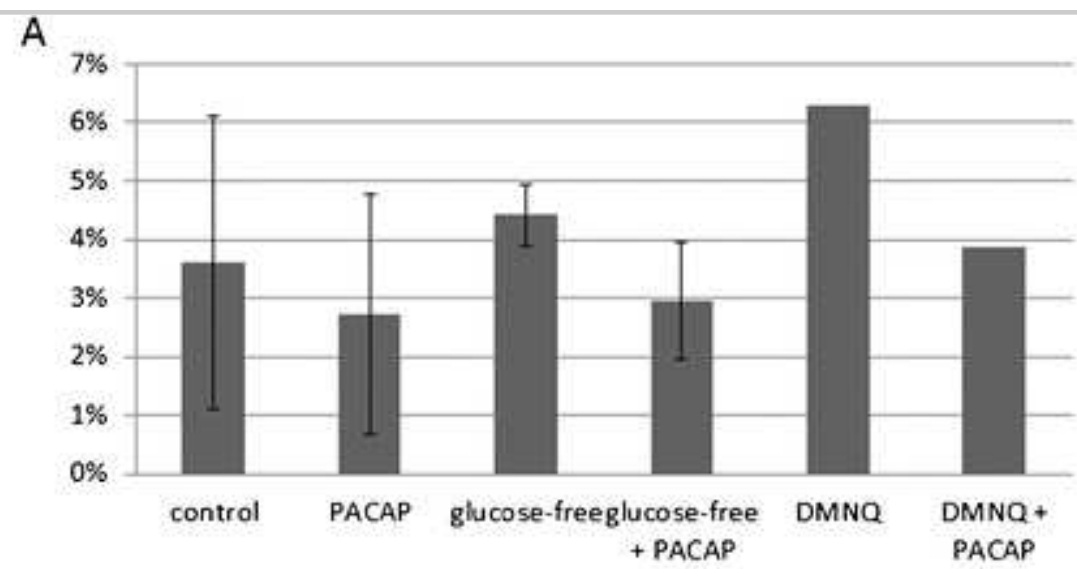

\section{B}

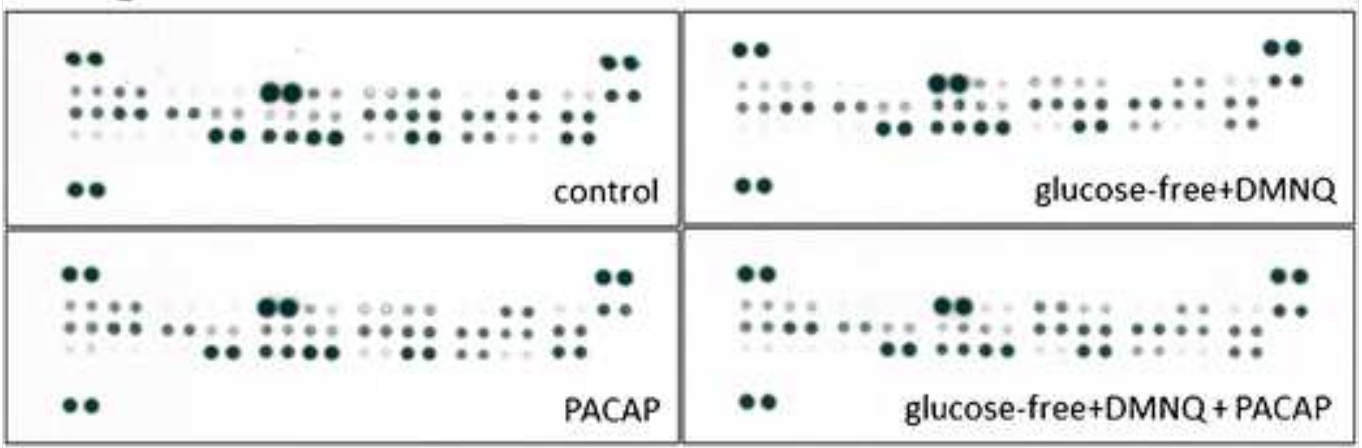

\begin{tabular}{|c|c|c|c|c|c|c|c|c|c|c|c|}
\hline tat. & & & & & & & & & & & tot. \\
\hline Bas & 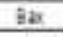 & 352 & $30-x$ & sts-cass? & ci. casp3 & casave & daA1 & chat-25 & covpin & Evatuith & ace \\
\hline Trail-1 & Trab? & Fast & 52 & Hiff 2 & $n 0-2$ & $N-2$ & Hes.7 & $\operatorname{Ris} 60$ & kero & $H \in A 2$ & livis: \\
\hline $\mathrm{PON} 2$ & 22 & 25 & 20.52 & 7.051 & P.053 & P. 520217 & $\sin \alpha$ & servivin & tw: 21 & TYAP & ovs. \\
\hline tet. & & & & & & & & & & & \\
\hline
\end{tabular}

(double spot each)

Effect of PACAP on the Junctional Complex of Brain Endothelial Cells

Our main goal, however, was to understand whether PACAP influenced the tight and adherens junctions of cerebral endothelial cells. Therefore, we measured the transendothelial electrical resistance (TEER), which is a very sensitive marker of the paracellular barrier. PACAP1-38 induced a marked 
increase in TEER when applied in the apical (upper) compartment, but no effect was observed when applied in the basolateral (lower) compartment (Fig. 3). The effect appeared immediately after administration: PACAP attenuated the medium change-induced TEER drop and maintained the TEER at higher levels in comparison to the control. When applied to both the apical and the basolateral side or when lower PACAP concentrations were administered (not shown), the effect was slighter and lasted for a shorter time.

\section{Fig. 3}

Effect of PACAP on the TEER of cerebral endothelial cells. RBECs were cultured on semipermeable filter inserts. PACAP was applied in a concentration of $100 \mathrm{nM}$ either in the upper, lower, or both $(\mathrm{u}+1)$ compartments. TEER was monitored using the cellZscope ${ }^{\circledR}$ system. Values are $\%$ control at start of the experiment, representing average \pm SD. ${ }^{*} p<0.05$ as assessed by ANOVA and Bonferroni's post hoc test (red indicates PACAP upper vs. control, violet indicates PACAP $\mathrm{u}+1$ vs. control). One representative of three independent experiments is presented

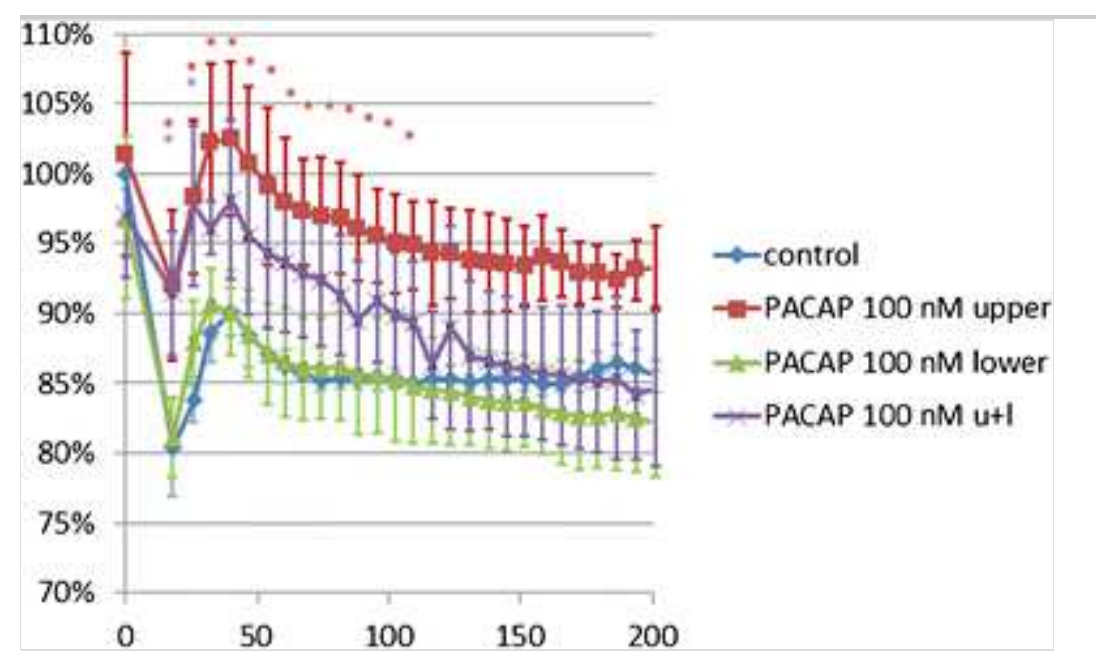

As a next step, we examined whether PACAP had a protective effect on the disassembly of the junctions induced by different pathological conditions. Glucose deprivation and DMNQ-induced oxidative stress alone or in combination induced the disruption of the continuous membrane staining of the junctional proteins ZO-1, VE-cadherin, occludin, and claudin-5 (Fig. 4 and Suppl. Fig. 2). In the presence of glucose deprivation or DMNQ-induced oxidative stress, we observed several holes on the membrane staining. When glucose deprivation and oxidative stress were applied concomitantly, we observed an almost total disappearance of the transmembrane junctional proteins from the cell membranes. Arrows in Fig. 4 and Suppl. Fig. 2 point to 
the discontinuities in the membrane staining. Transmembrane proteins (VE-cadherin, occludin, and claudin-5) were more sensitive to stress-induced disruption than the cytoplasmic protein ZO-1.

\section{Fig. 4}

a-d Effect of PACAP on the localization of tight and adherens junction proteins. RBECs or D3 cells were exposed to glucose deprivation- and/or DMNQ-induced oxidative stress in the absence or presence of $100 \mathrm{nM}$ PACAP. Immunofluorescence stainings were performed using anti-ZO-1 or anti-VEcadherin antibodies. Junctional disruption (indicated by arrows) is represented by the discontinuity or disappearance of the membrane staining. One representative of three independent experiments is presented 
A RBEC, ZO-1 staining

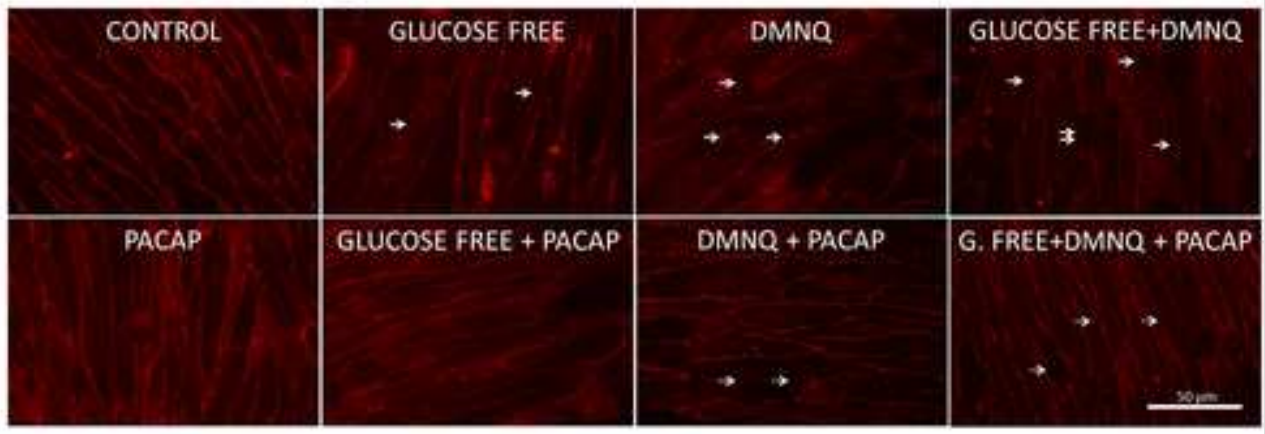

B RBEC, VE-cadherin staining

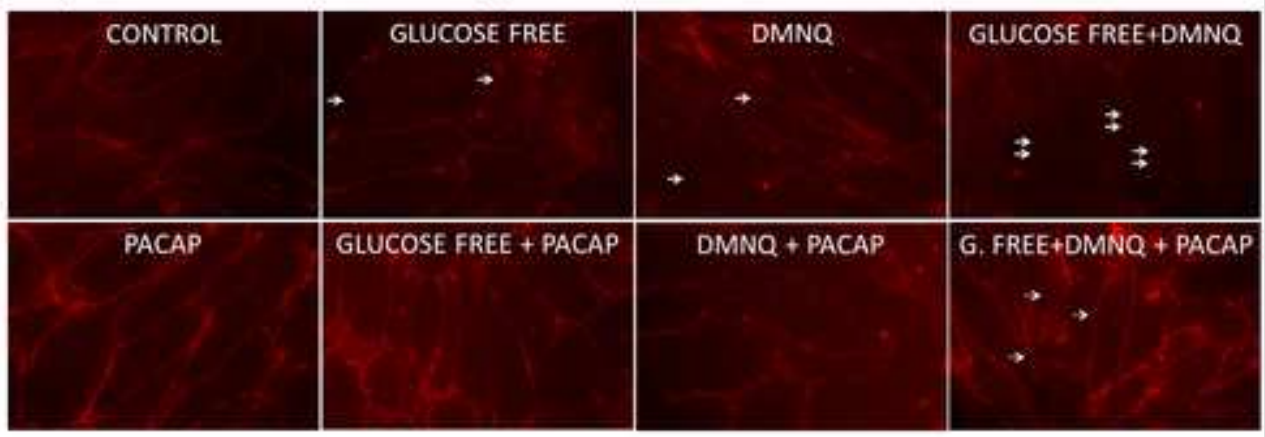

C D3, ZO-1 staining

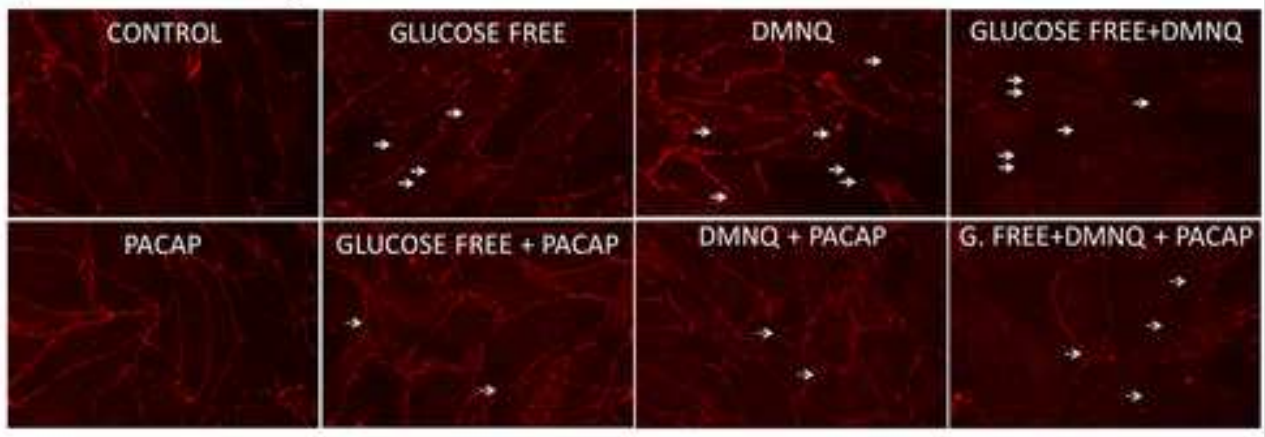

D D3, VE-cadherin staining

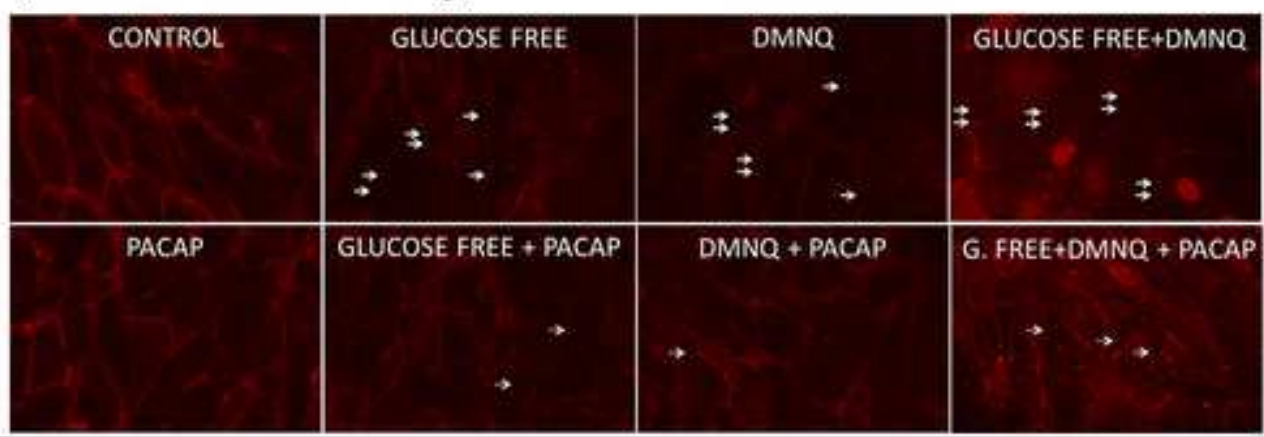

PACAP treatment alone induced a stronger junctional staining of claudin-5, occludin (Suppl. Fig. 2), ZO-1, and VE-cadherin (Fig. 4 ) in RBECs. The same effect was seen in D3 cells which do not form such a tight barrier as primary cells (Fig. 4). Moreover, PACAP had a protective effect on glucose deprivation- and/or DMNQ-induced junctional disassembly in both RBECs and D3 cells, i.e., no or less holes could be observed on the membrane stainings and the territories with absent membrane staining were much 
smaller. PACAP treatment could almost totally maintain the continuity of the tight and adherens junctions in glucose-free conditions in both brain endothelial cell types analyzed and could significantly improve the DMNQ-induced junctional disassembly. When glucose deprivation and oxidative stress were applied concomitantly, we observed an almost total disappearance of the transmembrane junctional proteins from the cell membranes and a severe disruption of the ZO-1 staining. PACAP could partly reverse these effects (Fig. 4).

To test whether PACAP influenced not only the localization but also the expression of the junctional proteins, we performed Western-blot experiments. Our results indicated that PACAP did not significantly induce the expression of tight and adherens junction proteins in RBECs or D3 cells (Fig. 5 and Suppl. Fig. 3). Only the amount of occludin increased in D3 cells, but not in the tight barrier forming RBECs. We observed a decrease in the amount of ZO-1 protein in response to glucose-free conditions combined with DMNQ; however, this was not affected by PACAP.

\section{Fig. 5}

Effect of PACAP on the expression of tight and adherens junction proteins. RBECs or D3 cells were exposed to glucose deprivation- and DMNQ-induced oxidative stress in the absence or presence of $100 \mathrm{nM}$ PACAP. Western-blot experiments were performed using the RIPA-soluble fractions. $\beta$-Actin was used as a loading control. One representative of three independent experiments is presented. Quantitative analysis is shown on Suppl. Fig. 3 


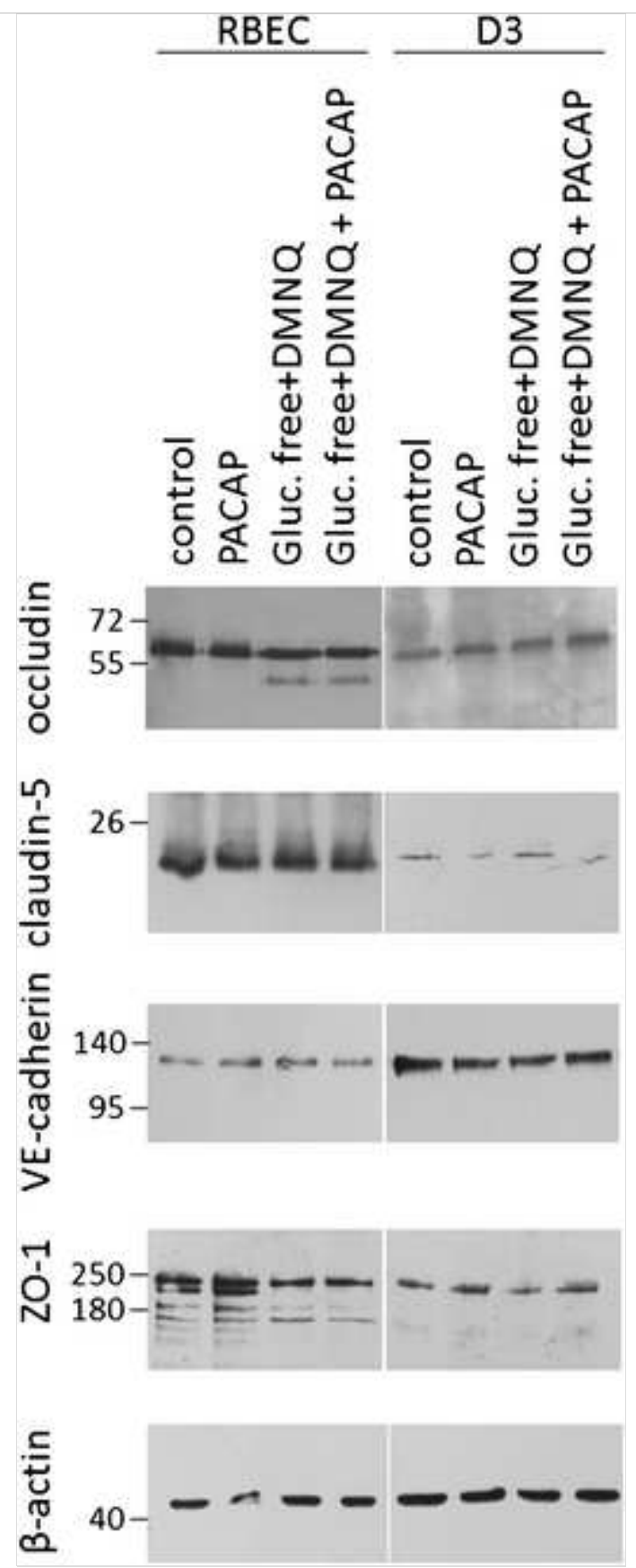

\section{Discussion}

Pituitary adenylate cyclase-activating polypeptide and its receptors have been shown to have widespread distributions in organism. However, the expression of PACAP and PACAP receptors on cells of the BBB has not been investigated so far, except for astrocytes, which have been shown to express PACAP, PAC1, VPAC1, and VPAC2 (Grimaldi and Cavallaro 1999; Jaworski 2000 ). In accordance with literature data, we showed the presence of PACAP and its three receptors on astrocytes. Additionally, we observed the presence of VPAC 2 and low amounts of PAC1 and VPAC1 in brain pericytes. We also showed that microvascular cerebral endothelial cells expressed PACAP. In accordance, isolated brain microvessels have been shown to release PACAP, 
which is decreasing with age (Tripathy et al. 2012).

We also detected VPAC2 in cerebral endothelial cells, while VPAC1 was present only in human, but not in rat brain endothelial cells. We have observed that PACAP treatment induced a marked increase in TEER when applied in the apical (upper) compartment. This suggests that PACAP receptors are probably present in the apical and not in the basolateral membrane. The two membrane parts are divided by the tight junctions which prevents the diffusion of proteins and lipids between the apical and the basolateral membranes. Interestingly, in the rat middle cerebral artery, all three PACAP receptors were found to be present and abluminal but not luminal VIP, PACAP-27, and PACAP-38 caused concentration-dependent arterial relaxations (Erdling et al. 2013), underlying the difference between macrovascular and BBB-forming microvascular brain endothelial cells.

According to our results, PAC1 was present neither in human nor in rat brain microvascular endothelial cells. Since most of the anti-apoptotic effects of PACAP are mediated through the PAC1 receptor (Seaborn et al. 2011), the absence of PAC1 from our cells may explain the lack of a significant pro-survival effect of PACAP on cerebral microvascular endothelial cells. Interestingly, although in response to glucose deprivation and oxidative stress marked cell death was observed (Suppl. Fig. 1A), this was not accompanied by the induction of apoptotic markers. This suggests that another type of cell death occurred (e.g., necrosis); however, we did not investigate in details the molecular processes leading to cell death. Nevertheless, glucose deprivationand/or oxidative stress-induced brain endothelial cell death was not reversed by the presence of PACAP.

The interaction of PACAP with the cerebral endothelium is far from being well-understood and mainly restricted to macrovessels. PACAP-27 and PACAP-38 were shown to be potent vasodilators in porcine neonatal pial vessels and rat middle cerebral artery (Lenti et al. 2007; Erdling et al. 2013). On the other hand, the effects of PACAP on microvascular brain endothelial cells, which form the BBB, are not well-characterized. High concentrations of PACAP (10 nM-1 $\mu \mathrm{M})$ were shown to significantly inhibit the activity of the cyclooxygenase pathway in isolated cerebral microvessels (Kis et al. 1999). However, the effects of PACAP on the barrier properties of the brain endothelium have not been investigated so far.

Capillary endothelial cells of the brain parenchyma form a restrictive 
paracellular barrier by the presence of well-developed tight junctions. cAMP is known to increase the transendothelial electrical resistance (TEER), which is the most important marker of the tightness of the TJs (for review see:

Krizbai and Deli 2003). Intracellular cAMP is known to strengthen the barrier function of the cerebral endothelium (Stelzner et al. 1989; Rubin et al. 1991; Rubin and Staddon 1999; Ishizaki et al. 2003). Moreover, cAMP-elevating agents, like adrenomedullin or cilostazol have also been shown to stabilize the tight junctions of cerebral endothelial cells (Kis et al. 2001; Horai et al. 2013 ). Nevertheless, all three receptors of PACAP (PAC1, VPAC1, and VPAC2) signal mainly through the cAMP/PKA pathway. Hence, we have focused our investigations on the potential effects of PACAP on the TJs of cerebral endothelial cells.

Our results show that PACAP stabilizes the junctional proteins in the membrane of brain endothelial cells in both control and pathological conditions. cAMP has been shown to induce the expression and phosphorylation of claudin-5 through different mechanisms and to enhance signals of claudin-5, occludin, ZO-1, ZO-2, and VE-cadherin along cell boundaries (Ishizaki et al. 2003). PACAP had no effect on the expression of the junctional proteins; however, the membrane staining of several tight and adherens junction proteins increased significantly, suggesting that PACAP induces junctional proteins to localize to the intercellular contacts.

In summary, this is the first report that PACAP improves the function of the tight junctions of cerebral endothelial cells without affecting their survival.

\section{Acknowledgments}

This work was supported by grants from the Hungarian Research Fund (OTKA PD-100958, K-100807, K-104984), the National Development Agency (Hungary-Romania Cross-Border Co-operation Program 2007-2013: HURO/1101/173/2.2.1, the TÁMOP-4.2.2.A-11/1/KONV-2012-0024, and 4.2.2.A-11/1/KONV-2012-0052 projects), the PTE-MTA "Lendület" Program, NAP, and the Arimura Foundation. I. Wilhelm was supported by the János Bolyai Research Fellowship of the Hungarian Academy of Sciences (BO/00320/12/8). The research of C. Fazakas and A. Tamás was supported by the European Union and the State of Hungary, co-financed by the European Social Fund in the framework of TÁMOP-4.2.4.A/2-11/1-2012-0001 "National Excellence Program."

\section{Electronic Supplementary Material}


Below is the link to the electronic supplementary material.

\section{Supplementary Fig. 1}

Effect of PACAP on the survival of cerebral endothelial cells. D3 cells were exposed to glucose-deprivation and DMNQ-induced oxidative stress in the absence or presence of $100 \mathrm{nM}$ PACAP. A: Phase contrast images showing the morphological changes. B: Densitometric analysis of pro-caspase 3 and the pro-apoptotic proteins Bad, Bax and cleaved caspase 3 from the apoptosis array presented on Fig. 2b. (JPEG 88 kb)

High resolution image (TIFF $18790 \mathrm{~kb}$ )

\section{Supplementary Fig. 2}

Effect of PACAP on the localization of tight junction proteins. RBECs were exposed to glucose-deprivation and/or DMNQ-induced oxidative stress in the absence or presence of $100 \mathrm{nM}$ PACAP. Immunofluorescence stainings were performed using anti-occludin or anti-claudin-5 antibodies. Junctional disruption (indicated by arrows) is represented by the discontinuity or disappearance of the membrane staining. One representative of three independent experiments is presented. (JPEG $67 \mathrm{~kb}$ )

High resolution image (TIFF $18790 \mathrm{~kb}$ )

\section{Supplementary Fig. 3}

Effect of PACAP on the expression of tight and adherens junction proteins. RBECs or D3 cells were exposed to glucose-deprivation and DMNQ-induced oxidative stress in the absence or presence of $100 \mathrm{nM}$ PACAP. Western-blot experiments were performed using the RIPA-soluble fractions. Densitometric analysis of three independent experiments is presented. ${ }^{* *}: \mathrm{p}<0.05, *$ : $\mathrm{p}<0.01$ compared to control, \#\#: $\mathrm{p}<0.05$ compared to G-free + DMNQ, as assessed by 
ANOVA and Bonferroni's post hoc test. (JPEG $7 \mathrm{~kb}$ )

High resolution image (TIFF $2196 \mathrm{~kb}$ )

\section{References}

Abbott NJ, Patabendige AA, Dolman DE, Yusof SR, Begley DJ (2010) Structure and function of the blood-brain barrier. Neurobiol Dis 37:13-25

Banks WA, Kastin AJ, Komaki G, Arimura A (1993) Passage of pituitary adenylate cyclase activating polypeptide 1-27 and pituitary adenylate cyclase activating polypeptide1-38 across the blood-brain barrier. J Pharmacol Exp Ther 267:690-696

Basille M, Cartier D, Vaudry D et al (2006) Localization and characterization of pituitary adenylate cyclase-activating polypeptide receptors in the human cerebellum during development. J Comp Neurol 496:468-478

Brown D, Tamas A, Reglodi D, Tizabi Y (2013) PACAP protects against salsolinol-induced toxicity in dopaminergic SH-SY5Y cells: implication for Parkinson's disease. J Mol Neurosci 50:600-607

Castorina A, Giunta S, Mazzone V, Cardile V, D’Agata V (2010) Effects of PACAP and VIP on hyperglycemia-induced proliferation in murine microvascular endothelial cells. Peptides 31:2276-2283

Dogrukol-Ak D, Kumar VB, Ryerse JS et al (2009) Isolation of peptide transport system-6 from brain endothelial cells: therapeutic effects with antisense inhibition in Alzheimer and stroke models. J Cereb Blood Flow Metab 29:411-422

Erdling A, Sheykhzade M, Maddahi A, Bari F, Edvinsson L (2013) VIP/PACAP receptors in cerebral arteries of rat: characterization, localization and relation to intracellular calcium. Neuropeptides 47:85-92 
Giunta S, Castorina A, Bucolo C, Magro G, Drago F, D’Agata V (2012)

Early changes in pituitary adenylate cyclase-activating peptide, vasoactive intestinal peptide and related receptors expression in retina of streptozotocin-induced diabetic rats. Peptides 37:32-39

Grimaldi M, Cavallaro S (1999) Functional and molecular diversity of PACAP/VIP receptors in cortical neurons and type I astrocytes. Eur J Neurosci 11:2767-2772

Horai S, Nakagawa S, Tanaka K et al (2013) Cilostazol strengthens barrier integrity in brain endothelial cells. Cell Mol Neurobiol 33:291-307

Hutamekalin P, Farkas AE, Orbok A et al (2008) Effect of nicotine and polyaromtic hydrocarbons on cerebral endothelial cells. Cell Biol Int 32:198-209

Ishizaki T, Chiba H, Kojima T et al (2003) Cyclic AMP induces phosphorylation of claudin-5 immunoprecipitates and expression of claudin-5 gene in blood-brain-barrier endothelial cells via protein kinase A-dependent and -independent pathways. Exp Cell Res 290:275-288

Jaworski DM (2000) Expression of pituitary adenylate cyclase-activating polypeptide (PACAP) and the PACAP-selective receptor in cultured rat astrocytes, human brain tumors, and in response to acute intracranial injury. Cell Tissue Res 300:219-230

Kis B, Gaspar T, Mezei Z, Gecse A, Telegdy G (1999) Pituitary adenylate cyclase-activating polypeptide inhibits the cyclooxygenase pathway of rat cerebral microvessels. Acta Physiol Scand 167:43-47

Kis B, Deli MA, Kobayashi H et al (2001) Adrenomedullin regulates blood-brain barrier functions in vitro. Neuroreport 12:4139-4142

Krizbai IA, Deli MA (2003) Signalling pathways regulating the tight junction permeability in the blood-brain barrier. Cell Mol Biol (NoisyLe-Grand) 49:23-31

Lenti L, Domoki F, Kis D et al (2007) Pituitary adenylate cyclaseactivating polypeptide induces pial arteriolar vasodilation through cyclooxygenase-dependent and independent mechanisms in newborn pigs. 
Miyata A, Arimura A, Dahl RR et al (1989) Isolation of a novel 38 residue-hypothalamic polypeptide which stimulates adenylate cyclase in pituitary cells. Biochem Biophys Res Commun 164:567-574

Paolinelli R, Corada M, Ferrarini L et al (2013) Wnt activation of immortalized brain endothelial cells as a tool for generating a standardized model of the blood brain barrier in vitro. PLoS One 8:e70233

Racz B, Gasz B, Borsiczky B et al (2007) Protective effects of pituitary adenylate cyclase activating polypeptide in endothelial cells against oxidative stress-induced apoptosis. Gen Comp Endocrinol 153:115-123

Reglodi D, Kiss P, Szabadfi K et al (2012) PACAP is an endogenous protective factor-insights from PACAP-deficient mice. J Mol Neurosci 48:482-492

Rubin LL, Staddon JM (1999) The cell biology of the blood-brain barrier. Annu Rev Neurosci 22:11-28

Rubin LL, Hall DE, Porter S et al (1991) A cell culture model of the blood-brain barrier. J Cell Biol 115:1725-1735

Seaborn T, Masmoudi-Kouli O, Fournier A, Vaudry H, Vaudry D (2011) Protective effects of pituitary adenylate cyclase-activating polypeptide (PACAP) against apoptosis. Curr Pharm Des 17:204-214

Stelzner TJ, Weil JV, O'Brien RF (1989) Role of cyclic adenosine monophosphate in the induction of endothelial barrier properties. J Cell Physiol 139:157-166

Sziraki I, Erdo F, Beery E et al (2011) Quinidine as an ABCB1 probe for testing drug interactions at the blood-brain barrier: an in vitro in vivo correlation study. J Biomol Screen 16:886-894

Tripathy D, Sanchez A, Yin X, Martinez J, Grammas P (2012) Age-related decrease in cerebrovascular-derived neuroprotective proteins: effect of acetaminophen. Microvasc Res 84:278-285 
Tsuchikawa D, Nakamachi T, Tsuchida M et al (2012) Neuroprotective effect of endogenous pituitary adenylate cyclase-activating polypeptide on spinal cord injury. J Mol Neurosci 48:508-517

Vaudry D, Falluel-Morel A, Bourgault S et al (2009) Pituitary adenylate cyclase-activating polypeptide and its receptors: 20 years after the discovery. Pharmacol Rev 61:283-357

Weksler BB, Subileau EA, Perriere N et al (2005) Blood-brain barrierspecific properties of a human adult brain endothelial cell line. FASEB J 19:1872-1874

Wilhelm I, Farkas AE, Nagyoszi P et al (2007) Regulation of cerebral endothelial cell morphology by extracellular calcium. Phys Med Biol 52:6261-6274

Wilhelm I, Fazakas C, Krizbai IA (2011) In vitro models of the blood-brain barrier. Acta Neurobiol Exp (Wars) 71:113-128 